\title{
A Novel HandofF Decision Algorithm IN CAll Admission Control Strategy to UTILIZE THE SCARCE SPECTRUM IN WIRELESS MOBILE NETWORK
}

\author{
Alagu $\mathrm{S}^{1}$, Meyyappan $\mathrm{T}^{2}$ \\ ${ }^{1}$ Research Scholar, Department of Computer Science and Engineering \\ Alagappa University, Karaikudi, Tamilnadu, India \\ sivaalaguehotmail.com \\ ${ }^{2}$ Professor, Department of Computer Science and Engineering \\ Alagappa University, Karaikudi, TamilNadu, India \\ meyslotuseyahoo. com
}

\begin{abstract}
Wireless networking is becoming an increasingly important and popular way of providing global information access to users on the move. One of the main challenges for seamless mobility is the availability of simple and robust Channel Allocation algorithm. The radio propagation environment and related handoff challenges are different in cellular structures. A handoff algorithm with fixed parameters cannot perform well in different system environments. In this paper, motivated by the facts that the scarce spectrum should be utilized efficiently, a new Dynamic Channel allocation scheme is proposed. Performance of the proposed dynamic channel allocation scheme is compared with existing channel allocation schemes such as fixed channel, static guard channel and Guard Channel with Channel Borrowing. The experimental results reveal that the proposed algorithm outperforms traditional methods in bandwidth utilization, handoff dropping rate and new call blocking rate.
\end{abstract}

\section{KEYWORDS}

Handoff, Guard Channel, Call Admission, Call Blocking, NHDA, Spectrum

\section{INTRODUCTION}

Wireless data networks are usually composed of a wired, packet-switched, backbone network and one or more wireless (e.g., cellular radio or infrared) hops connecting mobile hosts to the wired part. The wireless part is organized into geographically-defined cells, with a control point called a base station (BS) for each of these cells which is depicted in Figure 1. The base stations are on the wired network and provide a gateway for communication between the wireless infrastructure and the backbone interconnect. As a mobile host $(\mathrm{MH})$ travels between wireless cells, the task of routing data between the wired network and the MH must be transferred to the new cell's base station. This process, known as a handoff, must maintain end-to-end connectivity in the dynamically reconfigured network topology. 


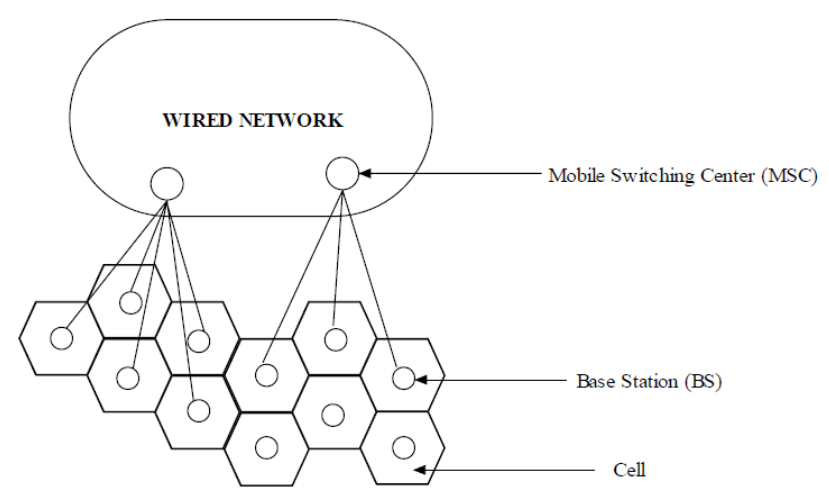

Figure 1. Cellular Architecture

\subsection{Mobile Communication System}

In modern cellular architecture there are limited available spectrums. The fixed base stations (BS) are interconnected to each other through a fixed network. They communicate with mobile stations (MS) via wireless links. The geographic area is divided into cells in which there is a base station serving each cell. Cells are divided into groups, in which each group is controlled by a mobile switching center (MSC). Neighboring cells overlap with each other to ensure the continuity of communications, when the users move from one cell to another. Fixed number of channels (spectrum) is assigned to each base station.

A channel in the system can be thought of as a fixed frequency bandwidth (FDMA), a specific time-slot within a frame (TDMA), or a particular code (CDMA), depending on the multiple access technique used. BSs and MSCs take the responsibility of allocating channel resources to mobile stations. Same set of channels is reused in another cell far apart enough so that the cochannel interference is negligible. The co-channel reuse distance is defined as the minimum distance at which channels can be reused with negligible interference [1].

\subsection{Allowing Mobility of a Subscriber}

In order to allow mobility to a subscriber, the cellular network has to have the ability to track down the subscriber when a call is made to them and should allow the subscriber to make calls while they are not in their home BS. Tracking down is only possible if the system maintains information about the location of the subscriber's Mobile device. Also the Mobile device should know the appropriate channels to await signals from the system. In order for both the cellular system and the Mobile device to have this required knowledge, there are two main procedures to follow when the subscriber turns on a Mobile device in any location. They are Searching for channels and Register to the nearest Base Station.

\subsubsection{Searching for channels}

There are two channels that are searched by the Mobile device which is depicted in Table 1 . This procedure enables the Mobile device to identify the correct channels to await signals from the system. 
Table 1 .Types of Channels

\begin{tabular}{|l|l|}
\hline Channel & Purpose \\
\hline $\begin{array}{l}\text { Strong Dedicated } \\
\text { Channel (DCC) }\end{array}$ & $\begin{array}{l}\text { A channel used for the } \\
\text { transmission of digital } \\
\text { control information from a } \\
\text { base station to the Mobile } \\
\text { device or vice versa. }\end{array}$ \\
\hline $\begin{array}{l}\text { Strong Paging } \\
\text { Channel }\end{array}$ & $\begin{array}{l}\text { A channel used by the MSC } \\
\text { for seeking the Mobile } \\
\text { device when a call made to } \\
\text { it. }\end{array}$ \\
\hline
\end{tabular}

\subsubsection{Register to the Nearest Base Station}

The Mobile device registers by sending MIN and ESN as shown in Table 2.

Table 2.Access Validation

\begin{tabular}{|l|l|}
\hline Access Code & Purpose \\
\hline Mobile Identification & $\begin{array}{l}\text { The telephone number of } \\
\text { the cellular instrument } \\
\text { assigned to the subscriber. }\end{array}$ \\
\hline $\begin{array}{l}\text { Electronic Serial } \\
\text { Number (ESN) }\end{array}$ & $\begin{array}{l}\text { This is assigned to the } \\
\text { Mobile device by the } \\
\text { manufacturer. }\end{array}$ \\
\hline
\end{tabular}

The MIN and ESN are used by the MSC for access validation. This involves checking with the information stored in the home base station of the subscriber. Information about the mobile device and its current position is stored in the Mobile Switching Centre (MSC) [2]. Both the information stored in the MSC and the paging channel is used by the MSC to direct any arriving call to the appropriate subscriber. When the subscriber makes calls, they are allowed to be anywhere within the network as this involves the Mobile device accessing base station, which could be located in any of the cells throughout the network. This base station can use the information it has retrieved from the home base station of the subscriber to direct their calls.

During a call, the base station would monitor the signal level from the Mobile device. When the Mobile device is moved into a new cell, the signal level will fall to a critical value causing the base station to inform the MSC about this event. The MSC would instruct the entire surrounding base stations to measure the Mobile device's signal level and transfer the control to the base station receiving the strongest signal level [4]. This is known as hand-over or hand off and occurs within $400 \mathrm{~ms}$. The subscribers are hardly aware of the break in signal. Now registration is done with the new BS. Location information stored in the MSC about this mobile device is updated. If the mobile device is moved into a cell belonging to a different cluster it would also have to register with the new MSC.

\section{HANDOFF}

Mobile Networks have gained an impulsion in the past few years in rapacious dimensions [3]. And since then mobility becomes a distinct feature of wireless mobile cellular system [5]. While a call (mobile caller/user in service) is in progress the channel (frequency, time slot, spreading code, or combination of them) associated with the current connection is changed through Channel Allocation Control (CAC) proposals [6]. The existing call may change its present Base Station (BS) also termed as Mobile Terminal (MT) to a new one. This phenomenon is whatever we call handover (handoff). It is shown in Figure 2. 
Usually, this handover mechanism supports continuous services by transfer of an ongoing call from the current cell to the next adjacent cell as the mobile (MS) moves through the coverage area. Either crossing a cell boundary of current BS by mobile station (MS) or deterioration in quality of the signal in the current channel is the primary responsible factor for initiating a handover [6][5].

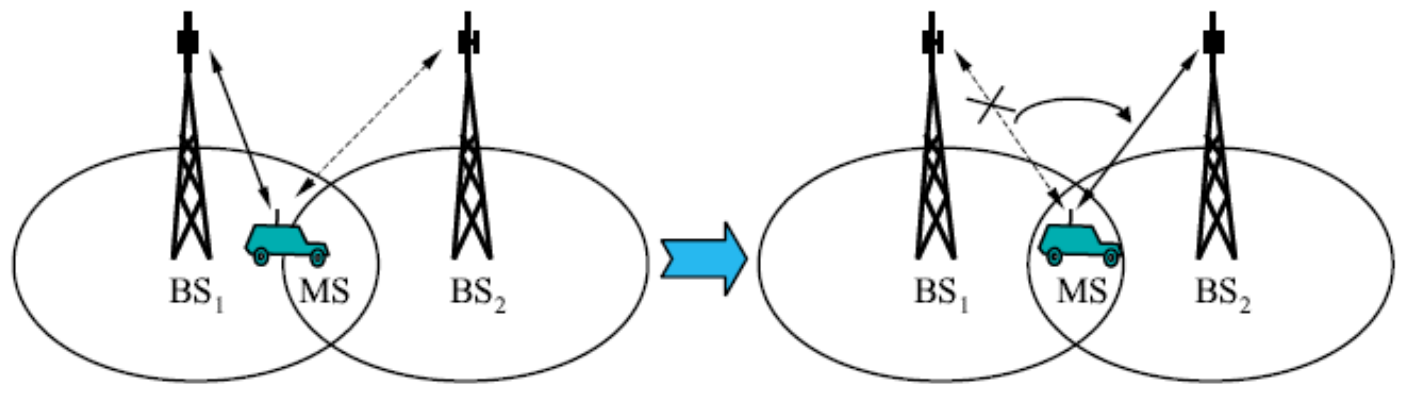

Figure 2. Handoff Scenario

A successful handoff provides continuation of the call which is vital for the perceived quality of service (QoS). In case the next cell does not have a radio channel available for the incoming MS, handoff blocking occurs and the call is dropped. The lack of channel resources also results in the blocking of new calls. The universally accepted design concept in cellular networks is that blocking of handoff requests is less desirable than the blocking of new calls. The QoS is mainly determined by the two blocking probabilities and the overall resource utilities. One of the important objectives in the development of the new generation is improving the quality of cellular service, with handoffs nearly invisible to the MSs.

Network protocols in cellular wireless data networks must update routes as a mobile host moves between cells. Most current handoff schemes in wireless networks result in data loss or large variations in packet delivery times. Unfortunately, many applications, such as real-time multimedia applications and reliable transport protocols, adapt to long term estimates of end-toend delay and loss. Violations of these estimates caused by handoff processing often result in degraded performance. For example, loss during handoff adversely affects TCP performance and high packet loss and variable delays result in poor real-time performance.

\subsection{Channel Allocation}

In wireless mobile networks, the service area is divided into cells each of which is equipped with a number of channels. New originating calls in the cell coverage area and the handoff calls are sharing these channels. When any of these calls arrives at a cell where channel is not available, it has to be blocked or queued or rejected depending on the call admission control schemes. The probability of the new originating call in the cell that is rejected is called Call Rejection probability and the probability that a handoff call rejected is called Handoff Rejection Probability.

Generally the Handoff request is initiated either by the Mobile Station or by the Base Station. Different types of handoff decision protocols are used in various cellular systems. Some of them are:

\section{- Network Controlled Handoff}

In this scheme, the Mobile Switching Center (MSC) is responsible for the overall handoff decision [1]. MSC measures the signal strength and receivers threshold from different Base Stations. It then decides on the handoff request to a Base Station whose signal level is closest [1][14]. 
- Mobile Assisted Handoff

Here the Mobile Station (MS) is responsible for finding the Base Station (BS) whose signal strength is closest to it. The MS measures the signal strengths periodically in the neighboring BS. Based on the received measurements, the BSs and MSC decides when to handoff [9][14].

\section{- Mobile controlled Handoff}

In this scheme the MS has got the full control in handoff decision. Both BS and MS measures the signal strength in the neighboring BSs and the current BS sends them to the MS. The MS decides when to handoff based on the information gained from the BS and itself [9][10][14].

\subsection{Performance Metrics for Handoffs}

The following are considered as the performance metrics for handover of calls from one cell coverage area to the others.

- Call blocking probability: The probability that a new call attempt is blocked.

- Handoff blocking probability: The probability that a handoff attempt is blocked.

- Handoff probability: The probability that while communicating with a particular cell, an ongoing call requires a handoff before the call terminates. This metric is translated to obtain the average number of handoffs per cell.

- Call dropping probability: The probability that a call terminates due to handoff failure. This metric can be derived directly from the handoff blocking probability and the handoff probability.

- Rate of handoff: The number of handoff per unit time.

- Duration of interruption: The length of time during handoff for which the mobile terminal is in communication with neither base station.

- Delay: The distance that the mobile user moves from the point at which, the handoff should occur to the point at which it does.

\section{LITERATURE SURVEY}

Many papers in the literature of related work addresses the categorization of the schemes Based on Guard channel concept. In the cellular network, channel assignment strategies can be classified into fixed, flexible and dynamic [7].The existing literature addresses the Static Guard Channel allocation exclusively for handoff and fixed channel system where there are no separate guard channels exclusively for handoff. In fixed channel assignment (FCA) scheme, fixed numbers of channels are assigned to each cell and there isn't any Guard Channel set aside exclusively for handoff requests. Whenever new call request or handoff request arrives, the base station will check to see if there is a channel available in current cell. The call will be connected if there is a channel available and it will be dropped if there isn't any channel left. So handoff request and new call request are dealt with equally. The cell doesn't consider the difference between Handoff request and new call request. It assigns the channels to BS by First Come First Serve basis [5][12][13]. The Quality of Service is not satisfied because the handoff blocking rate is as same as new call blocking rate. The so called "Guard-channel" (GC) concept offers a means of improving the probability of a successful handoff by reserving a certain number of channels allocated exclusively for handoff requests. The remaining channels can be shared equally between handoff requests and new calls [1][9]. Allocating Guard channels for Handoff improves the overall throughput which was discussed in our previous papers [12][13]. If the guard channel number is too big, the new call blocking rate will be high because several channels are set aside for handoff requests even when the traffic load is low. In this case, the resources are wasted by not serving either for handoff request or new call request. If the number 
is too small, the handoff blocking rate can't be guaranteed under high traffic load. So this scheme enhances the QoS by reducing the handoff blocking rate in a stable traffic load. While when the traffic load is changing periodically or dynamically due to big event or working rush hours, it is not flexible enough to get good QoS. This scheme sometimes ends up with inefficient spectrum utilization. Careful estimation of channel occupancy time distributions is essential in order to minimize this risk by determining the optimum number of guard channels. Static Guard Channel Allocation with Channel Borrowing scheme [15] does improves the channel utilization but the complexity of the algorithm increases since preemption is done in every allocation.

\section{Proposed Work}

In this paper, the authors devise a scheme - A Novel Handoff Decision Algorithm in Call Admission Control Strategy (NHDA). In this proposed new scheme, the channels for handoff requests are dynamically allocated based on the handoff failure probability observed for a certain past period in the network. This scheme aims to utilize the scarce spectrum efficiently and also to balance the load in the network traffic. The new call dropping rate determines the fraction of new calls that are rejected. The handoff blocking rate is closely related to the fraction of admitted calls that terminate prematurely due to handoff. Limited channels, a scarce resource, should be utilized effectively. Efficient resource utilization is the main objective of this research work. For effective resource utilization, less number of Guard channels should be assigned to the handoff calls during low traffic load in the network. If more channels are reserved for the handoff request in this condition, the resources are wasted as the channels serve neither for handoff request nor a new call request. On the other hand, if the number of handoff request is more than the number of available Guard channels, then the number of guard channels should be increased. The balance of the new call rejection rate and handoff call rejection rate are monitored and maintained to get better resource utilization in cellular network. The Channel allocation model is shown in Figure 3.

A call being forced to terminate during the service is more annoying than a call being blocked at its start. Hence the handoff call blocking probability is much more stringent than new call blocking probability. Therefore it is intuitively clear that priority is given to handoff requests by assigning GCh channels exclusively for handoff calls among the $\mathrm{C}$ channels in a cell. The remaining Oc $(=\mathbf{C}-\mathbf{G C h})$ channels are shared by both originating calls and handoff requests. The selection of number of guard channels exclusively for handoff call is essentially important factor to get good Quality of Service. In the proposed scheme the guard channel GCh is initially assigned and dynamically altered based on the traffic in the network.

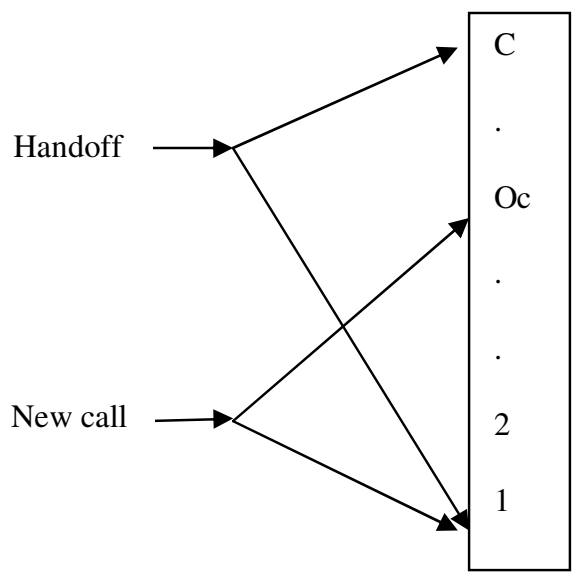

Figure 3. Channel allocation model with priority for handoff calls 
International Journal of Wireless \& Mobile Networks (IJWMN) Vol. 4, No. 6, December 2012

\subsection{Call Admission Control}

Quality of Service (QoS) provisioning in wireless networks is a challenging problem due to the scarcity of wireless resources, i.e. radio channels, and the mobility of users. Call Admission Control (CAC) is a fundamental mechanism used for QoS provisioning in a network. It restricts the access to the network based on resource availability in order to prevent network congestion and service degradation for already supported users. A new call request is accepted if there are enough idle resources to meet the QoS requirements of the new call without violating the QoS for already accepted calls. With respect to the layered network architecture, different QoS parameters are involved at different layers. At physical layer, bit-level QoS parameters such as bit energy-to-noise density describe the quality of service [3]. In packet-based communication systems, packet-level QoS parameters such as packet loss, delay and jitter characterize the perceived quality of service [3]. However, most of the existing research on call admission control in cellular networks has focused on an abstract representation of the network in which only call-level QoS parameters, namely, call blocking and dropping probabilities are considered. When a mobile terminal (mobile user) requests service, it may either be granted or denied service. This denial of service is known as call blocking, and its probability as call blocking probability $(\mathrm{Pb})$. An active terminal in a cellular network may move from one cell to another. The continuity of service to the mobile terminal in the new cell requires a successful handoff from the previous cell to the new cell. A handoff is successful if the required resources are available and allocated for the mobile terminal. The probability of a handoff failure is called handoff failure probability (Pf ). During the life of a call, a mobile user may cross several cell boundaries and hence may require several successful handoffs. Failure to get a successful handoff at any cell in the path forces the network to discontinue service to the user. This is known as call dropping or forced termination of the call and the probability of such an event are known as call dropping probability (Pd). In general, dropping a call in progress is considered to have a more negative impact from the user's perspective than blocking a newly requested call.

According to the above definition, the call dropping probability, $\mathrm{Pd}$, and handoff failure probability, Pf, are different parameters. While the handoff failure probability is an important parameter for network management, the probability of call dropping (forced termination) may be more relevant to mobile users and service providers. Despite this fact, most research papers focus on the handoff failure probability because calculating Pf is more convenient.

\subsection{Channel Assignment Schemes}

Channels are managed at each cell by channel assignment schemes based on co-channel reuse constraints [6]. In Static Guard Channel Allocation scheme, a set of channels is permanently assigned to each base station. A new call can only be served if there is a free channel available in the cell. Due to non-uniform traffic distribution among cells, Static Guard Channel Allocation schemes suffer from low channel utilization. Channel borrowing scheme overcomes this problem at the expense of increased complexity and signaling overhead. In this research work the authors have proposed the Novel Handoff Decision Algorithm in Call Admission Control Strategy (NHDA) where the channels are allocated dynamically based on the observation in the traffic.

\subsection{Novel Handoff Decision Algorithm (NHDA)}

The selection of number of guard channels exclusively for handoff call is essentially important factor to get good Quality of Service. For different type of traffic load and mobility factor, different number of guard channels is needed to be allocated. The number of guard channels can't be fixed when the traffic load is changing with the time. The authors address this problem through the proposed scheme NHDA. 
The proposed scheme automatically searches the optimal number of Guard Channels to be reserved for handoff calls at each BS. In this paper the author considers for a Base Station BS, having total number of channels $\mathrm{C}$, the Guard channels exclusively for handoff will be GCh. The rest of the available channels are used by the new originating calls in that cell and also by the handoff calls, which is say Co. A new call request will be granted for admission if the total number of on-going calls (including handoff calls from other cells) is less than the number Co. A handoff call request will be granted for admission if the total number of on-going calls in the cell is less than the total capacity C.

The algorithm NHDA can be illustrated as follows:

\section{Data Structure}

Consider the following parameters in a particular BS coverage area,

The total number of available channels $-\mathrm{C}$

Open Access Channels (new calls + Handoff calls) - Co

Guard channels for handoff calls

Where, $\mathrm{C}=\mathrm{Co}+\mathrm{GCh}, \mathrm{Co}=\mathrm{C}-\mathrm{Gch}$ and $\mathrm{GCh}$ is allocated dynamically

$\mathrm{Oc}=$ number of on-going calls

$\mathrm{Nc}=$ number of admitted new originating calls

$\mathrm{Hc}=$ number of admitted handoff calls

$\mathrm{H}=$ Total number of handoff call (admitted+rejected)

Where, $\mathrm{Oc}=\mathrm{Nc}+\mathrm{Hc}$

$\mathrm{Pd}=$ Call dropping probability // used in FCA scheme

$\mathrm{Pf}=$ Probability of Handoff failure

$\mathrm{Pb}=$ Call blocking probability

$\mathrm{t}=$ time period

$\mathrm{Th}=$ Threshold for handoff call rejection probability

Algorithm: NHDA (t, C) // the algorithm takes time period and channels as input

\{

$\mathrm{Co}=\mathrm{C}-\mathrm{GCh}$

For every handoff call request Do

\{

If $\mathrm{Oc}<\mathrm{C}$, then

\{

$\mathrm{Hc}=\mathrm{Hc}+1$ and grant admission

$\mathrm{Oc}=\mathrm{Oc}+1$

\}

Otherwise, $\mathrm{Pf}=\mathrm{Pf}+1$ and reject.

\}

For every new call request Do

\{

If $\mathrm{Oc}<\mathrm{Co}$, then

\{

$\mathrm{Nc}=\mathrm{Nc}+1$ and grant admission

$\mathrm{Oc}=\mathrm{Oc}+1$

\}

\}

Otherwise, $\mathrm{Pd}=\mathrm{Pd}+1$ and reject.

If a call is completed or handoff to another cell

\{ 
$\mathrm{Oc}=\mathrm{Oc}-1$

Check with MSC whether the ended call is handoff call or new originated call

If handoff call then $\mathrm{Hc}=\mathrm{Hc}-1$

Else $\mathrm{Nc}=\mathrm{Nc}-1$

\}

If a handoff call is dropped and $\mathrm{Pf} / \mathrm{H}>=$ AuTh then

\{

$\mathrm{GCh}=\min \{\mathrm{GCh}+1, \mathrm{Cmax}\}$

If $\mathrm{Pf} / \mathrm{H}<=\mathrm{AdTh}$ for $\mathrm{N}$ consecutive handoff calls, then

$\mathrm{GCh}=\max \{\mathrm{GCh}-1, \mathrm{Cmin}\}$

\}

$\mathrm{Nc}$ and $\mathrm{Hc}$ are reported to understand the successful handoff and new calls at a specified time period.

\}//end of the algorithm: NHDA

As the number of Guard Channels allotted plays a vital role to the key performance, it is dynamically altered every specific time period say t. In this approach the number of guard channels which is to be allocated is determined through optimizing certain performance goal with service quality constraints. When a base station experiences high handoff blocking rate, the number of guard channels will be increased until the handoff blocking rate drops to below its threshold. When a base station does not get to use a significant portion of the guard channels over a period of time, the number of guard channels is gradually decreased until most of the guard channels are used frequently. By doing this, the handoff blocking rate is controlled to close to its threshold.

The proposed algorithm increases the number of guard channels GCh, when a handoff call is dropped under the condition that $\mathrm{Pf} / \mathrm{H}>=\mathrm{Au}^{*} \mathrm{Th}$, and it decreases the number of guard channels after a number of consecutive handoff calls under the condition that $\mathrm{Pf} / \mathrm{H}<=\mathrm{Ad} * \mathrm{Th}$. $\mathrm{Au}$ and $\mathrm{Ad}$ are usually chosen to be less than 1 . By choosing $\mathrm{Au}<1$, the algorithm will most likely keep the handoff blocking rate below its given threshold.

The simulation studies are performed for comparisons of the proposed algorithm with fixed channel allocation (FCA) and static guard channel allocation policy. The result proves that the algorithm guarantees that the handoff dropping rate is below its given threshold and at the same time the new call dropping rate is minimized.

\section{EXPERIMENTAL RESULTS}

In the simulation study of the NHDA, the authors used a model that adhered to the general assumptions made in the literature. Below, the authors describe the various components of the simulation model and the assumptions for these components.

\subsection{Cell Model}

In the simulation, the authors use 2-D cellular system model with wrap around which is depicted in figure 4. Our simulation tests use a $6 * 6$ cellular patch with wrap around, a cell radius of $1000 \mathrm{~m}$, a minimum reuse distance of 3 , and a TD equal to 0.8 of the cell radius. MSs are allowed to wrap around to the other side of the system when moves out of system boundary. It eliminates the burden of handling out of bound situations and is considered an efficient way to approximate the simulation of a very large cellular system. Each cell is considered as a circle and has exactly six neighbors. Object oriented approach is used to implement the real world environment. Figure 4 shows the cell structure considered for simulation. 


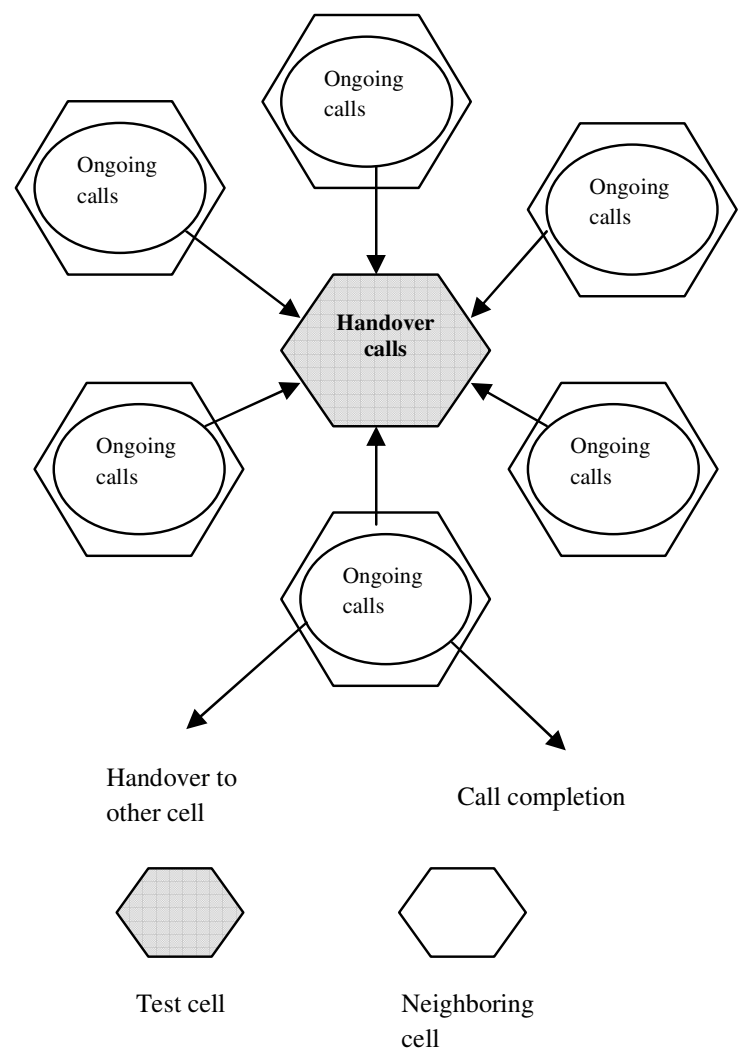

Figure 4. Simulation model for a single Cell

\subsection{Traffic Model}

The authors use exponential distribution to determine the duration of each generated call with a mean of 180s. New Calls arrive according to a Poisson process and homogeneous traffic among all cell were considered. Each cell is assigned 20 channels. The traffic load to each cell is defined as:

$$
\text { Arrival Rate to the cell * Average call duration } * 100
$$

Number of channels per cell

\subsection{Mobility Model}

In our model, each MS is assigned initial speed and direction with an average speed of 18 meters/s and a maximum speed of 24 meters/s. After a specified time period, which is generated randomly, the speed and direction of the MS are updated.

The selection of ideal time period is an important parameter too. If the time period is too small, the overhead in network is too high and the algorithm can't take much advantage. If the time period is too large, the handoff blocking rate will be adjusted to fit to the changing traffic load too slowly and as a result the QoS will not be guaranteed. The authors have chosen 2 hours time period to test and simulate the scenario.

\subsection{Simulation results}

Extensive simulations are conducted to evaluate the performance of NHDA scheme. 
The result comprises of comparison between the 4 schemes as Handoff Handling without using Guard Channels-FCA, Using static Guard Channels, using Guard Channel with Channel borrowing scheme, and the proposed scheme NHDA. The parameters chosen for simulation are:

busy_channels : Number of channels occupied by calls.

next_event_type : Type of next event New call, New Handoff, Channel release.

total_calls : Number of calls generated in or handed to the cell.

new_success : New calls which have been assigned channels by the BS.

ho_success : Handovers which have been assigned channels by the BS

ho_fail : Handovers which have not been assigned channels.

Blocked : New calls which have not been allocated channels.

incell_success : New incell call or handover which have been assigned channels.

incell_blocked : New incell call or handover which have not been assigned channels.

incluster_success : New incluster call or handover which have been assigned channels.

incluster_blocked: New incluster call or handover which have not been assigned channels.

outcluster_success: New outcluster call or handover which have been assigned channels.

outcluster_blocked: New outcluster call or handover which have not been assigned channels.

call_type : Type of call; Incell, Incluster, Outcluster

BTS_index : Index of Base Station whose event will occur.

Capacity : Load which a backbone link can handle.

next_call : Time at which next new call will be generated.

next_event_time : Time at which next event will occur.

next_handover : Time at which next new handover will be generated.

ho_delay : Time for which a handover is stored in the handover queue.

miat : Mean inter arrival time. Time difference between successive calls

hmiat : Handoff Mean inter arrival time. Time difference between successive handover.

$\mathrm{Oc}=0, \mathrm{Nc}=0, \mathrm{Hc}=0, \mathrm{~Pb}=0, \mathrm{Pf}=0, \mathrm{H}=0$

$\mathrm{t}=20$ seconds // time period for updating the measurements

$\mathrm{C}=20 / /$ No. of channels

$\mathrm{GCh}=10 / /$ Guard Channels exclusively for Handoffs

$\mathrm{Au}=0.9$

$\mathrm{An}=0.6$

$\mathrm{Th}=0.8$

$\mathrm{N}=10 / / \mathrm{No}$. of consecutive calls

Number of cells: 36

Position measurement interval: $3 \mathrm{sec}$.

Mean call duration: $180 \mathrm{sec}$

Average speed of an Ms: $18 \mathrm{~m} / \mathrm{s}$

Simulation time: $200,000 \mathrm{sec}$.

Number of Radio Channels $=50$ per cell.

Number of Local Link Channels $=50$ per cell.

Average time for a new call $=60 \mathrm{sec}$.

Average time for a handover call $=45 \mathrm{sec}$.

Maximum handover queue time $=15 \mathrm{sec}$.

Capacity of Backbone Links $=75$ calls.

Formulae Involved:

Call Blocking =

Total number of calls blocked

Total number of calls processed 
Total no. of handoffs not assigned Channels

Handoff failures $=$

Total number of calls processed.

$$
\mathrm{TSC}+\mathrm{TSH}
$$

Throughput $=$

Total number of calls processed

Where,

TSC $=$ Total number of calls that have been assigned channels and backbone links.

$\mathrm{TSH}=$ Total number of handoffs that have been assigned channels and backbone links.

The following graphs show the comparative study of the four schemes, Fixed Channel Assignment without using Guard Channels, Static Guard channel assignment, Guard Channel assignment with Channel borrowing scheme and the proposed scheme NHDA.

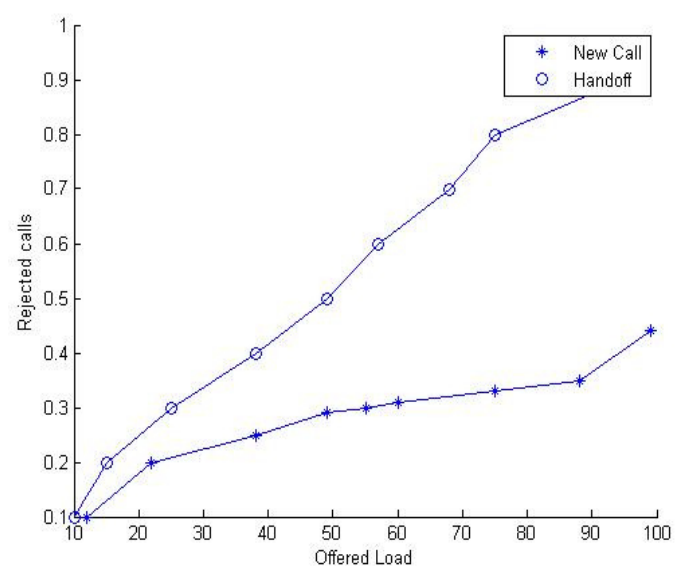

Figure 5. New Call handling and Handoff call handling without using Guard Channels (FCA)

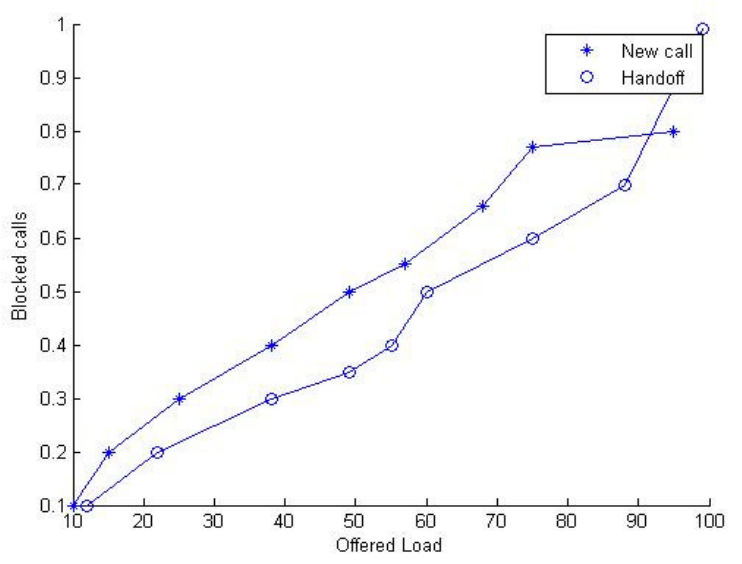

Figure 6. Static allocation of Guard Channels exclusively for Handoffs 


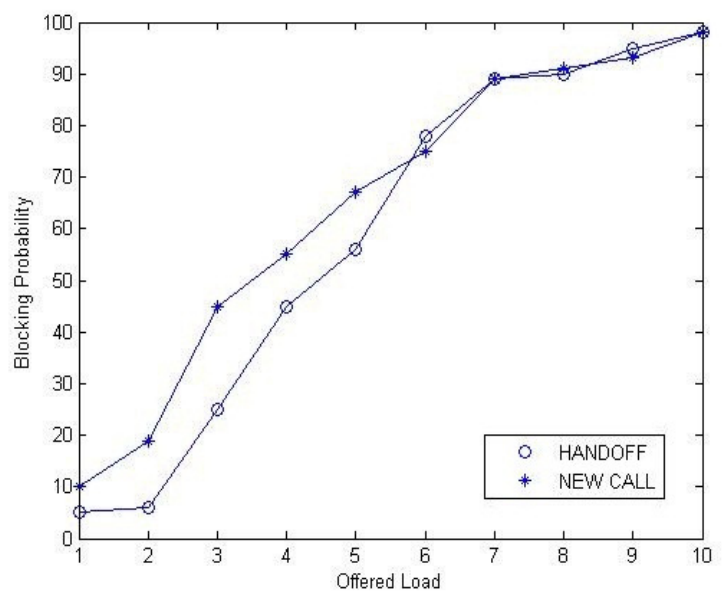

Figure 7. Guard Channel Allocation with Channel Borrowing Scheme

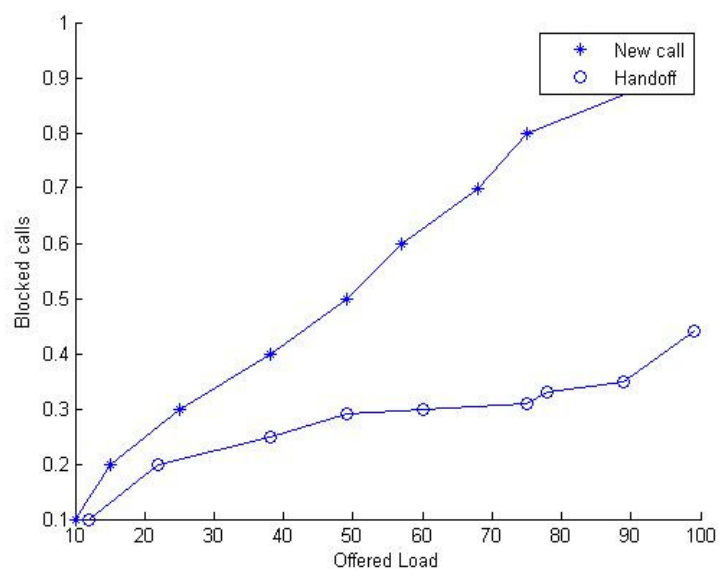

Figure 8. Novel Handoff Decision scheme

Figure 5 shows the simulated output of the FCA scheme where no guard channels are allocated for handoff calls. It simply works in FCFS (First Come First Served) manner. The output graph shows that the Handoff Call rejection rate is slightly high than the new originating call rejection rate. But it should be noted here that handoff calls should be given higher priority than the new originating calls. The overall performance is also not satisfactory since both new call rejection and handoff call rejection ratio is comparatively high.

Figure 6 shows the simulated output of the static guard channel allocation scheme i.e., fixed number of guard channels exclusively allocated for handoff. Here the number of handoff call rejection is reduced but the new call rejection is highly increased because the number of guard channels allocated is high than which is actually required. Moreover in some cases if the number of guard channels is less, then handoff rejection rate will increase and hence affect the throughput.

Figure 7 shows the simulated output of the Guard Channel Allocation with Channel Borrowing scheme. The graph shows that the handoff blocking and new call blocking varies often as borrowing strategy is implemented. Here the handoff blocking rate is reduced but the 
complexity of the algorithm increases and as a result of that the overall throughput is not satisfactory.

Figure 8 shows the simulated output of our proposed scheme - Novel Handoff Decision Algorithm (NHDA). Here as the channels are not allocated static and they are allocated based on the traffic in the past certain period of time. The number of guard channels gets dynamically adjusted and it is clearly seen from the graph that both new calls and handoff calls utilizes the channel properly and the call rejection rate is low for both. Hence there is tradeoff.

\section{CONCLuSion}

This paper presents a new algorithm for call admission control in a mobile cellular network. From the graph it is obvious that the new strategy shows better resource utilization.

Due to the unique characteristics of mobile cellular networks, mainly mobility and limited resources, the wireless resource management problem has received tremendous attention. As a result, a large body of work has been done extending earlier work in fixed channel allocation by introducing new techniques. In this paper, the authors presented a Novel Handoff Decision Algorithm in call admission control strategy for cellular networks. This new scheme utilizes the available resources effectively. In this work, a significant contribution has been made in the area of call admission control with the hope of improving the Call Admission Control performance.

For evaluating the performance of this scheme, a simulation model was created and lots of tests were done. The simulation result shows that the proposed algorithm can adapt to the changes in traffic conditions (changes in the call arrival rate) and can achieve optimal performance in terms of guaranteeing handoff call blocking threshold and at the same time minimizing the new call blocking rate. This adaptive approach can automatically search the optimal number of guard channels to be reserved at a base station. Existing Guard channel allocation schemes lack dynamism to cope up with dynamic network traffic. The proposed algorithm adjusts the number of guard channels dynamically according to the dropping rate of handoff calls in a certain period of time. It either increases or decreases the number of guard channels allocated based on observed handoff rejection threshold. The proposed scheme NHDA possesses high degree of spectrum utilization with good QoS and is a simple algorithm with a satisfied implementation complexity.

\section{REFERENCES}

[1] Yi Zhang and Derong Liu, "An Adaptive Algorithm for Call Admission Control in Wireless Networks", IEEE 2001

[2] Qing-an-Zeng and Dharma P.Agarwal, "Handbook of Wireless Networks and Mobile Computing”,John wiley \& sons, Inc., 2002

[3] Kishore S Trivedi et.al "Analytical Modeling of Handoffs in Wireless cellular networks", Elsevier Information Sciences, 2002

[4] A. Iera, A. Molinaro and S. Marano, "Handoff Management with Mobility Estimation in Hierarchical Systems", IEEE Transactions on Vehicular Technology, vol. 51, Sept. 2002.

[5] I.Ramani and S.Savage. SyncScan "Practical fast handoff for 802.11 Infrastructure Networks", Proceedings of IEEE INFOCOM, March 2005

[6] Nasif Ekiz, Tara Salih, Sibel Kucukoner and Kemal Fidanboylu, "An overview of Handoff techniques in Cellular Networks", World Academy of Science, Engineering and Technology, 62005

[7] F. Siddiqui and S. Zeadally, "Mobility Management across Hybrid Wireless Networks: Trends and Challenges," Computer Communications, vol. 29, no. 9, pp. 1363-1385, May 2006.

[8] Nasser, N., Hasswa, A. and Hassanein, "Handoffs in Fourth Generation Heterogeneous Networks", IEEE Communications Magazine, vol. 44, pp. 96-103, 2006. 
International Journal of Wireless \& Mobile Networks (IJWMN) Vol. 4, No. 6, December 2012

[9] Rami Tawil, Jaques Demergian, Guy Pujolle, "A Trusted Handoff Decision Scheme for the Next Generation Wireless Networks”, IJCSNS International Journal of Computer Science and Network Security, VOL.8 No.6, June 2008

[10] Yoo, S.-J.,et al., "Analysis of fast handover mechanisms for hierarchical mobile IPv6 network mobility”, Wireless Personal Communications, 48(2), 215-238., 2009.

[11] Asd Malak Z. Habeib, Hussein A. Elsayed, Salwa H. Elramly and Magdy M. Ibrahim, "Heterogenous Networks Handover Decision Triggering Algorithm Based on Measurements Messages Transfer using IP Option Header", The Seventh International Conference on Wireless and Mobile Communications - ICWMC 2011

[12] Alagu.S and Meyyappan.T, "Analysis of Algorithms for Handling Handoffs in Wireless Mobile Networks”, International Journal of P2P Network Trends and Technology- Vol1Issue2, 2011.

[13] Alagu S and Meyyappan T, “Analysis of Handoff Schemes in Wireless Mobile Network”, IJCES International Journal of Computer Engineering Science, Vol1 Issue2, Nov. 2011.

[14] V. S. Kolate, G. I. Patil, A. S. Bhide, "Call Admission Control Schemes and Handoff Prioritization in $3 G$ Wireless Mobile Networks", International Journal of Engineering and Innovative Technology (IJEIT) Vol1, Issue3, March 2012

[15] Alagu.S and Meyyappan.T, "Guard Channel Allocation with Channel Borrowing Scheme to handle Handoffs in Wireless Mobile Networks", Proceedings of the National Conference on Computer Applications, Bharathiar University, March 2012.

\section{Authors}

Mrs.S.Alagu, M.Sc., M.Phil., currently, Ph.D. Research Scholar in Department of Computer Science and Engineering, Alagappa University, Karaikudi. She has a teaching experience of 10 years. She has published research papers in National and International Journals and Conferences. Her research area includes Wireless Mobile Networks.

Dr. T. Meyyappan M.Sc., M.Phil., M.B.A., Ph.D., currently, Professor, Department of Computer Science and Engineering, Alagappa University, Karaikudi, TamilNadu. He has obtained his Ph.D. in Computer Science in January 2011 and published a number of research papers in National and International journals and conferences. He has been honored with Best Citizens of India Award 2012 by International Publishing House, New Delhi. He has developed Software packages for Examination, Admission Processing and official Website of Alagappa University. His research areas include Operational Research, Digital Image Processing, Fault Tolerant computing, Network security and Data Mining. 